Publ. RIMS, Kyoto Univ.

19 (1983), 729-741

\title{
Abstract Twisted Duality for Quantum Free Fermi Fields
}

\author{
By \\ Jerzy J. FoIT*
}

\begin{abstract}
Using the properties of standard subspaces of the 1-particle space for the free Fermi field we prove the twisted duality for the von Neumann algebras associated with real closed subspaces. This is done by application of the Tomita-Takesaki theory.
\end{abstract}

\section{Introduction}

Locality is one of the fundamental principles of quantum field theory. It means that observables which are localized in spacelike separated regions must commute. Duality strengthens this by claiming that observables commuting with all observables localized in the spacelike complement $\mathcal{O}^{\prime}$ of $\mathcal{O}$ must themselves be localized in $\mathcal{O}$. Expressing this in terms of local von Neumann algebras $\mathfrak{A}(\Theta)$ generated by the observables localized in $\mathcal{O}$, we have $\mathfrak{U}(\mathcal{O})=\mathfrak{U}\left(\mathcal{O}^{\prime}\right)^{\prime}$. $\mathfrak{A}\left(\mathcal{O}^{\prime}\right)^{\prime}$ denotes the commutant of $\mathfrak{A}\left(\mathcal{O}^{\prime}\right)$. Duality plays an important role in the theory of superselection rule [1], [2], so that it is important to know for which models duality holds.

The first step is to check this property for free field theories. Indeed, the results for interacting theories [13], [14] are restricted to two space-time dimensions and rely on the corresponding properties for free fields. The proof of duality for free Bose fields was first given by Araki and falls naturally into two parts. Araki showed in [3] that the commutant of the von Neumann algebra $R(M)$ generated by the Weyl operators based on a closed real subspace $M$ of the 1 -particle space is $R\left(M^{\prime}\right)$ where $M^{\prime}$ denotes the symplectic complement of $M$. This

Communicated by H. Araki, September 27, 1982.

* Universität Osnabrück, Fachbereich 4, D-4500 Osnabrück, West Germany 
proof was later simplified by Eckmann and Osterwalder [5] using TomitaTakesaki theory. They identified the antiunitary involution $J$ associated with the faithful vacuum state of $R(M)$ as the second quantization of an antiunitary involution $j$ on 1-particle space. It was simplified further in [6] by identifying the antilinear involution $S$ as the second quantization of an antilinear involution $s$ on 1-particle space. Other versions of this part of the proof were given by Dell'Antonio [8] using infinite tensor products and by Rieffel [7] using an abstract commutation theorem.

The second part of Araki's proof [4] identified the closed real subspace $M(\mathcal{O})$ associated with the space-time region $\mathcal{O}$ for the free Bose scalar field and showed for a suitably large class of regions that $M(\theta)^{\prime}$ $=M\left(\Theta^{\prime}\right)$.

This paper is concerned with the analogue for Fermi fields of the abstract first part of Araki's proof. Of course, the concept of duality has to be modified to allow for the anticommuting nature of the underlying Fermi fields. The appropriate concept, twisted duality, was introduced in [1] where it was shown to imply duality for the underlying algebra of observables and proved for free Fermi fields on the basis of the results of [8].

Here we use the ideas of [6] to show that if $R(M)$ is the von Neumann algebra generated by the Fermi fields based on a closed real subspace $M$ of the 1-particle space then

$$
R(M)^{t^{\prime}}=R\left(i M^{\prime}\right)
$$

where $R(M)^{t}$ results from $R(M)$ by applying a Klein transformation.

\section{$\S 1$. Real Subspaces of a Complex Hilbert Space}

M. Rieffel and A. van Daele [9] study the properties of standard subspaces and their canonical involution in connection with Tomita-Takesaki theory. This was exploited in [6] to simplify the proof of duality for free Bose fields. For the reader's convenience, this section gives some definitions and propositions of [6] which are used later in this paper. 
Definition 1. 1. Let $H$ be a complex Hilbert space with $h, k \rightarrow(h, k)$ as scalar product, $M$ a subset of $H$.

$$
M^{\prime}=\{k \in H: \operatorname{Im}(h, k)=0, h \in M\}
$$

is the symplectic complement of $M$.

Proposition 1.2. Let $M$ be a subset of H. Then:

i) $M^{\prime}$ is a closed real subspace of $H$.

ii) If $M \subset N$, then $N^{\prime} \subset M^{\prime}$.

iii) $M^{\prime \prime}$ is the closed real subspace generated by $M$.

iv) $(M+i M)^{\prime}=M^{\prime} \cap i M^{\prime}=M^{\perp}$.

v) $M^{\prime}=\{0\}$ if $M$ is dense in $H$.

vi) For $M$ a closed real subspace of $H$ and $P$ an orthogonal projection in $H$, the following are equivalent:

$$
\begin{aligned}
& P M \subset M, \\
& (\mathbb{1}-P) M \subset M, \\
& P M^{\prime} \subset M^{\prime}, \\
& (\mathbb{1}-P) M^{\prime} \subset M^{\prime} .
\end{aligned}
$$

If one of these conditions is true, then

$$
P M^{\prime}=(P M)^{\prime} \cap P H \text {. }
$$

Definition 1.3. A real closed subspace $M$ in $H$ is said to be standard if $M \cap i M=\{0\}$ and $\overline{M+i M}=H$. If $M$ is standard, the map

$$
s(h+i k)=h-i k, \quad h, k \in M
$$

is said to be the canonical involution of $M$.

The following proposition gives a relationship between the properties of the operator $s$ and some structures of real subspaces. This connection is important for the proof of twisted duality.

Proposition 1.4. Let $M$ be standard. Then:

i) The canonical involution $s$ of $M$ is a densely defined, closed 
antilinear involution.

ii) $M^{\prime}$ is standard and the canonical involution of $M^{\prime}$ is $s^{*}$.

iii) If $s=j \delta^{1 / 2}$ is the polar decomposition of $s$, then $j^{2}=1, j \delta^{1 / 2}$ $=\delta^{-1 / 2} j$ and $j(M)=M^{\prime}$.

iv) Let $P$ be an orthogonal projection in $H$ such that $P M \subset M$, then $P M$ (resp. $(\mathbb{1}-P) M)$ is standard in PH $($ resp. $(\mathbb{1}-P) H)$.

The following proposition shows that it suffices to study standard real subspaces.

Proposition 1.5. Let $M$ be a real closed subspace of $H$. Let $P_{1}$ (resp. $P_{2}$ ) be the orthogonal projections on the complex subspaces orthogonal to $M$ (resp. to $\left.M^{\prime}\right)$. Then:

i) $\quad P_{1} P_{2}=0$ and, consequently, $P_{3}=1-P_{1}-P_{2}$ is an orthogonal projection in $H$.

ii) $P_{1} M=\{0\}, P_{1} M^{\prime}=P_{1} H$.

iii) $P_{2} M^{\prime}=\{0\}, P_{2} M=P_{2} H$.

iv) $P_{3} M$ is standard in $P_{3} H$.

v) $P_{3} M^{\prime}=\left(P_{3} M\right)^{\prime} \cap P_{3} H$.

\section{§ 2. The von Neumann Algebras Associated with Real Subspaces}

Definition 2. 1 . Let $M \subset H$ be a real closed subspace of $H$. Let $\psi(h)$ be the Fermi field on the Fock space $\Gamma(H)$ defined in the appendix. $R(M)=\{\psi(h): h \in M\}^{\prime \prime}$ is the von Neumann algebra generated by $\psi(h)$.

The generators $\psi(h)$ of $R(M)$ satisfy anticommutation relations in contrast to Bose fields. Therefore, we must modify the notion of duality for the field algebra. That is why we define a transformation called twist [1].

First we consider an automorphism on $R(M)$ separating Fermi and Bose structure in $R(M)$. The transformation $\psi(h) \rightarrow-\psi(h)$ extends to a unique automorphism $\gamma$ on $R(M)$ : 


$$
\begin{array}{lll}
r(F)=F & \text { for } & F \in R_{+}(M), \\
r(F)=-F & \text { for } & F \in R_{-}(M) .
\end{array}
$$

$R_{+}(M)$ is generated by even products of $\psi(h), R_{-}(M)$ is generated by odd products of $\psi(h)$.

Because of the invariance of the Fock state under $\gamma$ there exists a unitary operator $U$ on $\Gamma(H) \quad([10], 2.12 .11)$ having the following properties :

$$
\begin{aligned}
& U \Omega=\Omega, \\
& \gamma(F)=U F U^{*}, F \in R(M), U^{*}=U^{-1}=U .
\end{aligned}
$$

For the free field considered here we can choose $U=e^{i \pi N}$, where $N$ is the particle number operator.

By using $\gamma$ each $F \in R(M)$ can be written as

$$
F=F_{+}+F_{-} .
$$

$F_{+}, F_{-}$are defined as follows:

$$
\begin{aligned}
& F_{+}=\frac{1}{2}(F+\gamma(F)), \\
& F_{-}=\frac{1}{2}(F-\gamma(F)) .
\end{aligned}
$$

Definition 2.2. For the $\gamma$-invariant algebras the twisted algebra $R(M)^{t}$ is given by:

$$
R(M)^{t}=\left\{F_{+}+i F_{-} U: F \in R(M)\right\} .
$$

Proposition 2.3. i) $F \rightarrow F_{+}+i F_{-} U$ defines an isomorphism of $R(M)$ on $R(M)^{t}$ which is implemented by the unitary operator $V=\frac{1}{\sqrt{2}}(\mathbf{1}-i U)$, i.e.

$$
V F V^{*}=F_{+}+i F_{-} U
$$

ii) $\quad R(M)^{t t}=R(M)$.

iii) $R(M)^{\prime t}=R(M)^{t \prime}$.

iv) $R\left(i M^{\prime}\right) \subset R(M)^{t^{\prime}}$ (twisted locality).

$R(M)^{\prime}$ denotes the commutant of $R(M)$. 
Proof. i) is proved by a simple calculation.

ii) follows from the equation $V^{2} F V^{* 2}=F_{+}-F_{-}$.

iii) is a conseqence of the unitary of $V$.

iv) Let $F=F_{+}+F_{-} \in R(M)$ and $F^{\prime}=F^{\prime}{ }_{+}+F^{\prime}-\in R\left(i M^{\prime}\right)$. The terms of the sums fulfill the following relations:

$$
\begin{aligned}
& {\left[F_{+}, F_{+}^{\prime}\right]=0,} \\
& {\left[F_{+}, F_{-}^{\prime}\right]=0,} \\
& {\left[F_{-}, F_{-}^{\prime}\right]_{+}=0,}
\end{aligned}
$$

i.e.

$$
\left[F^{\prime}, V F V^{*}\right]=0
$$

and therefore, we have

$$
R\left(i M^{\prime}\right) \subset R(M)^{t^{\prime}}
$$

Theorem 2.4. Let $M_{\alpha} \subset H, \alpha \in I$, be real closed subspaces of H. Let $R\left(M_{\alpha}\right)$ be the von Neumann algebra associated with $M_{\alpha}$. $R(M)$ gives an isomorphism between the lattice of real subspaces and the sublattice of von Neumann algebras. Namely:

i) $R\left(M_{1}\right) \supset R\left(M_{2}\right)$ iff $M_{1} \supset M_{2}$.

ii) $R\left(M_{1}\right)=R\left(M_{2}\right)$ iff $M_{1}=M_{2}$.

iii) Let $\bigvee_{\alpha} M_{\alpha}=\overline{\sum_{\alpha} M_{\alpha}}$ be the closure of the set of all finite real linear combinations. Then we have:

$$
R\left(\bigvee_{\alpha} M_{\alpha}\right)=\bigvee_{\alpha} R\left(M_{\alpha}\right)
$$

iv) $R\left(\bigcap_{\alpha} M_{\alpha}\right)=\bigwedge_{\alpha} R\left(M_{\alpha}\right)$.

Our main thesis is

v) $R(M)^{\prime t}=R\left(i M^{\prime}\right)$ (twisted duality).

Proof. i) If $M_{1} \supset M_{2}$ we see immediately that $R\left(M_{1}\right) \supset R\left(M_{2}\right)$. Let $R\left(M_{1}\right) \supset R\left(M_{2}\right)$ and $M_{1} \not M_{2}$. From Proposition 1.2.ii) we have $i M_{2}^{\prime} \not i M_{1}^{\prime}$, i.e. there exists $h_{1} \in i M_{1}^{\prime}$ and $h_{1} \notin i M_{2}^{\prime}$. Moreover there is a vector $h_{2} \in M_{2}$ such that

$$
\operatorname{Re}\left(h_{1}, h_{2}\right)=\left[\psi\left(h_{1}\right), \psi\left(h_{2}\right)\right]_{+} \neq 0 .
$$

Consequently, we have: 


$$
\psi\left(h_{1}\right) \notin R\left(M_{2}\right)^{t^{\prime}}
$$

and

$$
\psi\left(h_{1}\right) \in R\left(M_{1}\right)^{t^{\prime}} .
$$

From this we get the relation $R\left(M_{1}\right)^{t^{\prime}} \not \subset R\left(M_{2}\right)^{t^{\prime}}$ which gives $R\left(M_{2}\right)$ $\not \subset R\left(M_{1}\right)$.

ii) follows from i).

iii) is a consequence of $i$ ), the linearity and the continuity with respect to the norm of $h \rightarrow \psi(h)$.

iv) follows from iii) and $v$ ).

v) remains to be proven and we first reduce to the case that $M$ is standard.

By Proposition 1.5 we have the following decomposition for $H$ :

$$
H=\bigoplus_{i=1}^{3} P_{i} H
$$

This decomposition induces an isomorphism between the von Neumann algebras on $\Gamma(H)$ and the von Neumann algebras on $\bigotimes_{i=1}^{3} \Gamma\left(P_{i} H\right)$ given by a unitary operator $T$ from $\Gamma(H)$ onto $\bigotimes_{i=1}^{3} \Gamma\left(P_{i} H\right)$ such that

$$
\begin{aligned}
& T \Omega=\bigotimes_{i=1}^{3} \Omega_{i}, \Omega_{i} \in \Gamma\left(P_{i} H\right), \\
& T \psi(h) T^{*}=\psi(h) \otimes U_{2} \otimes U_{3} \quad \text { for } \quad h \in P_{1} H, \\
& T \psi(h) T^{*}=\mathbb{1} \otimes \psi(h) \otimes \mathbb{1} \quad \text { for } h \in P_{2} H, \\
& T \psi(h) T^{*}=\mathbb{1} \otimes U_{2} \otimes \psi(h) \quad \text { for } \quad h \in P_{3} H, \\
& T U T^{*}=\bigotimes_{i=1}^{3} U_{i}, U_{i} \Omega_{i}=\Omega_{i}, U_{i}^{*}=U_{i}^{-1}=U_{i}, \\
& {\left[\psi\left(h_{i}\right), U_{i}\right]_{+}=0 \quad \text { for } h_{i} \in P_{i} H \text { and } i=1,2,3 .}
\end{aligned}
$$

For the algebras $R(M)$ and $R\left(i M^{\prime}\right)^{t}$ we get:

$$
R(M)=C \otimes \mathscr{B}\left(P_{2} H\right) \otimes R\left(P_{3} M\right)
$$

and

$$
R\left(i M^{\prime}\right)^{t}=\mathscr{B}\left(P_{1} H\right) \otimes C \otimes R\left(i P_{3} M^{\prime}\right)^{t} .
$$

$\mathscr{B}(H)$ is the set of all bounded operators on $H$. For the proof of duality we need the following results: 
Proposition 2.5. Let $H$ be a complex Hilbert space, $M$ a real closed subspace of $H$.

i) $\Omega \in \Gamma(H)$ is a cyclic vector for $R(M)$ if and only if $M$ $+i M$ is dense in $H$.

ii) $\Omega$ is a separating vector for $R(M)$ if and only if $M \cap i M$ $=\{0\}$.

Proof. i) Assume that $M+i M$ is not dense in $H$. The orthogonal complement of $M$ is, according to Proposition 1.2, equal to $M^{\perp}=(M+i M)^{\prime} \neq 0$. For $0 \neq h \in M^{\perp}$ and $k_{i} \in M, i=1,2, \cdots$ we have $\left(\psi(h) \Omega, \prod_{i} \psi\left(k_{i}\right) \Omega\right)=0$. This is in contradiction to the assumption that $\Omega$ is a cyclic vector for $R(M)$. Let now be $\overline{M+i M}=H$. As a consequence of the remark (A.9) we have $R(M) \Omega \supset R_{0}(M) \Omega=\underset{n}{\oplus} \underset{n}{\Lambda}(M+i M)$. $R_{0}$ is the *-algebra generated by polynomials of $\psi(h)$. As $\underset{n}{\oplus} \Lambda_{n}(M$ $+i M)$ is dense in $\Gamma(H), \Omega$ is a cyclic vector for $R(M)$.

ii) Let $M \cap i M=\{0\} . \quad \Omega$ is a separating vector for $R(M)$ because of i) and because of twisted locality. Conversely, assume $\Omega$ to be separating and $M \cap i M \neq\{0\}$. There exists a vector $0 \neq h \in M \cap i M$ such that $\psi(h) \in R(M), \quad i \psi(i h) \in R(M)$ and $(\psi(h)+i \psi(i h))=0$, i.e. $\psi(h)=$ $-i \psi(i h)$. Now we have

$$
(h, h)=2 \psi(h) \psi(h)=-i \psi(h) \psi(i h)-i \psi(i h) \psi(h)=-i \operatorname{Re}(h, i h)
$$

which is only possible for $h=0$.

For $M$ standard in $H, \Omega$ is a cyclic and separating vector of $R(M)$. In that case, we can use the structure of the Tomita-Takesaki-Theorem [11]. Let us now consider the antilinear operator

$$
S_{0}: R(M) \Omega \rightarrow R(M) \Omega, S_{0} A \Omega=A^{*} \Omega, A \in R(M) .
$$

$S_{0}$ is densely defined and closable. The polar decomposition of the closure $\bar{S}_{0}=S=J \Delta^{1 / 2}$ gives the Tomita-Takesaki operator $J$ with the property $J R(M) J=R(M)^{\prime}$. Now we consider the connection of $S$ and $J$ with the operators $s$ and $j$.

Definition 2. 6. $\widetilde{S}:=e^{-i(\pi / 4)} V^{*} \Gamma(i s), \quad \Gamma(i s)=\bigoplus_{n=1}^{\infty} \Gamma_{n}(i s)$. 
$\Gamma_{n}(i s), n=1,2, \cdots$ is the closure of the operator $\bigotimes^{n}(i s)$ on $\underset{n}{\Lambda} D(s)$, ${ }_{n} D(s)$ is the set of all linear combinations of vectors of the type $h_{1} \wedge \cdots \wedge h_{n}, h_{j} \in D(s), j=1, \cdots, n$, and $D(s)$ is the domain of $s$.

Proposition 2. 7. If $s=j \delta^{1 / 2}$ is the polar decomposition of $s$, then $\Gamma(s)=\Gamma(j) \Gamma\left(\delta^{1 / 2}\right)$ is the polar decomposition of $\Gamma(s)$.

Proof. Easy [6].

Proposition 2.8. Let $R_{0}(M), M$ standard in $H$, be the *-algebra generated by polynomials of $\psi(h), h \in M$.

i) $R_{0}(M) \Omega$ is the core of $S$ and $\widetilde{S}$.

ii) $S_{\mid R_{0}(M) \Omega}=\widetilde{S}_{\mid R_{0}(M) \Omega}$.

Proof. i) $R_{0}(M)$ is a core for $S$ since $R_{0}(M)$ is *-strongly dense in $R(M)$ [12]. By (A. 9), $R_{0}(M) \Omega=\bigoplus_{n} \Lambda_{n}(M+i M) . \bigoplus_{n} \Lambda_{n}(M+i M)$ is a core for $\Gamma(i s)$ and hence for $\widetilde{S}$.

ii) We have to compare the effect of $S$ and $\widetilde{S}$ on $\psi\left(h_{1}\right) \cdots \psi\left(h_{n}\right) \Omega$, $h_{j} \in M, j=1, \cdots, n, n=1,2, \cdots$ with each other. We use formula (A.8). Obviously, we have

$$
V^{*} \Gamma(i s) A(k)^{*}=A(i s k)^{*} V \Gamma(i s), \quad k \in D(s) .
$$

Therefore, it is very easy to evaluate expressions of the type $\widetilde{S} A\left(h_{1}\right) * \ldots$ $A\left(h_{n}\right) * \Omega$. Because we have:

$$
\widetilde{S} \prod_{j=1}^{k}\left(h_{\pi(j)}, h_{\pi(j+k)}\right)=\prod_{j=1}^{k}\left(h_{\pi(j+k)}, h_{\pi(j)}\right) \widetilde{S}
$$

we must only evaluate:

$$
\begin{aligned}
\widetilde{S} \theta_{1,2, \cdots, n} & =\widetilde{S} A\left(h_{1}\right) * \cdots A\left(h_{n}\right) * \Omega, \quad n=1,2, \cdots \\
\widetilde{S} \theta_{1,2, \cdots, n} & =e^{-i(\pi / 4)} \frac{1}{\sqrt{2}}(\mathbf{1}+i U) \Gamma(i s) A\left(h_{1}\right)^{*} \cdots A\left(h_{n}\right)^{*} \Omega \\
& =i^{n} e^{-i(\pi / 4)} A\left(h_{1}\right) * \cdots A\left(h_{n}\right) * \frac{1}{\sqrt{2}}\left(1+(-1)^{n} i U\right) \Omega \\
& =i^{n} \exp \left(i \frac{\pi}{4}\left((-1)^{n}-1\right)\right) A\left(h_{1}\right)^{*} \cdots A\left(h_{n}\right)^{*} \Omega .
\end{aligned}
$$


The factor $i^{n} e^{i(\pi / 4)((-1) n-1)}$ generates a sign:

$$
i^{n} \exp \left(i \frac{\pi}{4}\left((-1)^{n}-1\right)\right)=\exp \left(i \pi\left(\frac{n}{2}+\frac{(-1)^{n}-1}{4}\right)\right)=(-1)^{n(n-1) / 2} .
$$

If we change the order of the factors in

$$
\theta_{1, \ldots, n}=(-1)^{n(n-1) / 2} \theta_{n, n-1, \cdots, 1}
$$

we will get

$$
\widetilde{S} \theta_{1, \ldots, n}=\theta_{n, n-1, \ldots, 1} .
$$

Applying the relation (A.8) the last equation gives:

$$
\widetilde{S} \psi\left(h_{1}\right) \cdots \psi\left(h_{n}\right) \Omega=\psi\left(h_{n}\right) \cdots \psi\left(h_{1}\right) \Omega=S \psi\left(h_{1}\right) \cdots \psi\left(h_{n}\right) \Omega .
$$

As the functor $\Gamma$ respects polar decompositions (Proposition 2.7) and $S=\widetilde{S}$ we know from the uniqueness of the polar decomposition that

$$
J=e^{-i(\pi / 4)} V^{*} \Gamma(i j), \Delta^{1 / 2}=\Gamma\left(\delta^{1 / 2}\right) .
$$

Now we are able to prove v) of Theorem 2.4. According to TomitaTakasaki theory the twisted commutant of $R(M)$ is equal to

$$
R(M)^{\prime t}=\operatorname{VJR}(M) J V^{*}
$$

i.e. $R(M)^{\prime t}$ is generated by

$$
V J \psi(h) J V^{*}=V V^{*} \Gamma(i j) \psi(h) V^{*} \Gamma(i j) V^{*}, \quad h \in M .
$$

If we use the relations

$$
\begin{aligned}
& \Gamma(i j) \psi(h)=\psi(i j h) \Gamma(i j), \\
& V^{*} \Gamma(i j)=\Gamma(i j) V
\end{aligned}
$$

and

$$
\Gamma(i j) \Gamma(i j)=\mathbf{1}
$$

we obtain

$$
V J \psi(h) J V^{*}=\psi(i j h) .
$$

This means that $R(M)^{\prime t}$ is generated by $\psi(i j h)$. On the other hand, $\psi(i j h)$ generates $R\left(i M^{\prime}\right)$ by Proposition 1.4. iii) i.e.

$$
R(M)^{\prime t}=R\left(i M^{\prime}\right)
$$

completing the proof of the theorem. 


\section{Appendix. Fock Representation of CAR}

Let $H$ be a complex Hilbert space, $\Gamma(H)=\bigoplus_{n=0} \Gamma_{n}(H)$ the Fock space on $H . \quad \Gamma_{n}(H)$ is the $n$-fold antisymmetric tensor product of $H$ with $\Gamma_{0}(H)=C$. For each $h \in H$ we define a creation operator $A(h)^{*}$ on $\Gamma(H)$,

$$
\begin{aligned}
& A(h) *: \Gamma_{n}(H) \rightarrow \Gamma_{n+1}(H), \\
& A(h) * k_{1} \wedge \cdots \wedge k_{n}=h \wedge k_{1} \wedge \cdots \wedge k_{n}, \\
& \Gamma_{n}(H) \ni k_{1} \wedge \cdots \wedge k_{n}=\frac{1}{\sqrt{n !}} \sum_{\pi \in S_{n}} \operatorname{sgn} \pi k_{\pi(1)} \otimes \cdots \otimes k_{\pi(n)}
\end{aligned}
$$

$S_{n}$ is the symmetric group of order $n$. The adjoint operator $A(h)$ is given by:

(A. 2) $\quad A(h) k_{1} \wedge \cdots \wedge k_{n}=\sum_{r}(-1)^{r+1}\left(h, k_{r}\right) k_{1} \wedge \cdots \wedge k_{r-1} \wedge k_{r+1} \wedge \cdots \wedge k_{n}$ $A(h) \Omega=0$,

where $\Omega \in \Gamma_{0}(H),\|\Omega\|=1$ is the Fock vacuum. The operators $A(k)$ * and $A(h), h, k \in H$ satisfy the CAR

(A. 3) $\left[A(h), A(k)^{*}\right]_{+}=A(h) A(k)^{*}+A(k)^{*} A(h)=(h, k)$,

$$
[A(h), A(k)]_{+}=\left[A(h)^{*}, A(k)^{*}\right]_{+}=0 \text {. }
$$

We define the following self-adjoint operators on $\Gamma(H)$ :

$$
\psi(h)=\frac{1}{\sqrt{2}}\left(A(h)^{*}+A(h)\right)
$$

These operators have the following properties:

$$
[\psi(h), \psi(k)]_{+}=\operatorname{Re}(h, k),
$$

$$
\left(\Omega, \psi\left(k_{1}\right) \cdots \psi\left(k_{2 n+1}\right) \Omega\right)=0 \text {, }
$$

(A. 7) $\quad\left(\Omega, \psi\left(k_{1}\right) \cdots \psi\left(k_{2 n}\right) \Omega\right)=2^{-n}(-1)^{n(n-1) / 2} \sum_{\pi} \operatorname{sgn} \pi \prod_{j=1}^{n}\left(k_{\pi(j)}, k_{\pi(j+n)}\right)$

where the sum is over all $\pi \in S_{2 n}$ satisfying

$$
\pi(1)<\cdots<\pi(n), \pi(j)<\pi(j+n), j=1, \cdots, n .
$$

By definition (A.4) and relation (A.3) every vector 


$$
\psi\left(h_{1}\right) \cdots \psi\left(h_{n}\right) \Omega, h_{j} \in H, j=1, \cdots, n, n=1,2, \cdots
$$

can be written as a linear combination of the vectors $A\left(h_{1}\right) * \cdots A\left(h_{k}\right) * \Omega$, $k \leq n$. There is for $n \geq 2$ :

$$
\text { (A. 8) } \begin{aligned}
2^{n / 2} \psi\left(h_{1}\right) \cdots \psi\left(h_{n}\right) \Omega= & \sum_{k=0}^{[n / 2]}(-1)^{k(k-1) / 2} \sum_{\pi \in S_{n}}^{k} \operatorname{sgn} \pi \prod_{j=1}^{k}\left(h_{\pi(j)}, h_{\pi(j+k)}\right) \\
& \times h_{\pi(2 k+1)} \wedge \cdots \wedge h_{\pi(n)}
\end{aligned}
$$

where $\sum_{\pi \in S_{n}}^{k}$ denotes the sum over all permutations $\pi \in S_{n}$ with

$$
\begin{aligned}
& \pi(j)<\pi(j+k), \quad j=1,2, \cdots, k ; \\
& \pi(1)<\pi(2)<\cdots<\pi(k) ; \\
& \pi(2 k+1)<\cdots<\pi(n) .
\end{aligned}
$$

Remark. Let $R_{0}(M)$ be as in Proposition 2.8. Then we have:

$$
R_{0}(M) \Omega=\bigoplus_{n} \Lambda_{n}(M+i M),
$$

where $\underset{n}{\Lambda}(M+i M)$ is the set of all finite linear combinations of vectors of the type

$$
k_{1} \wedge \cdots \wedge k_{n}, \quad k_{j} \in M+i M, \quad j=1, \cdots, n, \quad n=1,2, \cdots .
$$

Proof. From (A.8) we get

$$
R_{0}(M) \Omega \subset \underset{n}{\oplus_{n}} \Lambda_{(}(M+i M)
$$

The opposite inclusion can be shown by inverting (A.8) which is obviously possible by recursion.

\section{Acknowledgement}

I am deeply indebted to Professor J. E. Roberts for suggesting the problem to me and for many helpful discussions.

\section{References}

[1] Doplicher, S., Haag, R. and Roberts, J. E., Comm. Math. Phys., 13 (1969), 1-23.

[2] — Comm. Math. Phys., 15 (1969), 173-200.

[3] Araki, H., J. Math. Phys., 4 (1963), 1343-1362. 
[4] - J. Math. Phys., 5 (1964), 1-13.

[5] Eckmann, J. P. and Osterwalder, K., J. Func. Anal., 13 (1973), 1-12.

[6] Leyland, P., Roberts, J. E. and Testard, D., C. N. R. S. preprint CPT 78/P. 1016 (1978).

[7] Rieffel, M., Comm. Math. Phys., 39 (1974), 153-164.

[8] Dell'Antonio, G., Comm. Math. Phys., 9 (1968), 81-117.

[9] Rieffel, M. and Van Daele, A., Pacific J. Math., 69 (1977), 187-221.

[10] Dixmier, J., Les $C^{*}$-algèbres et leurs représentations, 2. ed., Gauthier-Villars, Paris, 1969.

[11] Takesaki, M., Lecture in Mathematics, 128, Springer-Verlag, Berlin, 1970.

[12] Bratteli, O. and Robinson, D., Operator Algebras and Quantum Statistical Mechanics I, Springer-Verlag, New York, 1979.

[13] Summers, S. J., Comm. Math. Phys., 86 (1982), 111-141.

[14] Driessler, W., Comm. Math. Phys., 70 (1979), 213-220. 
